\title{
Prospective comparison of conventional radiography, low-dose computed tomography and magnetic resonance imaging in monoclonal gammopathies
}

\author{
Jiri Minarika, Petra Krhovska ${ }^{\mathrm{a}}$, Jan Hrbek ${ }^{\mathrm{b}}$, Tomas Pikaa , Jaroslav Bacovsky ${ }^{\mathrm{a}}$, Miroslav Herman ${ }^{\mathrm{b}}$, Vlastimil Scudla ${ }^{\mathrm{a}}$
}

\begin{abstract}
Aims. We carried out a prospective study in order to identify the best imaging approach for patients with newly diagnosed multiple myeloma (MM) and monoclonal gammopathy of undetermined significance (MGUS).

Methods. We assessed the extent of myeloma bone disease (MBD) in 112 individuals - 84 patients with MM and 28 individuals with MGUS. For the detection of osteolytic involvement we used whole-body magnetic resonance imaging (WB-MRI), low-dose computed tomography (LD-CT) and conventional radiography (CR). Each method assessed the presence of osteolytic involvement, compressive fractures and extramedullary involvement in the following regions: skull, spine and chest, pelvis and humerus and femur. We compared the difference in the number and extent of osteolytic involvement, especially the findings in CR negative patients.

Results. Conventional radiography showed no superiority in any of the evaluated regions, and failed in the detection of extramedullary massess and spine involvement. WB-MRI was best at imaging the spine including extramedullary involvement, however, detection of osteolytic lesions of the skull was limited in comparison with both CR and LD-CT. Both WB-MRI and LD-CT were comparable in imaging of lesions of pelvis, humerus, femur and the presence of extramedullary masses. LD-CT showed superiority in detection of skull lesions but lower sensitivity in spine compared to WB-MRI. Conclusions. Our results confirm that relying solely on $C R$ in the diagnostics of $M M$ is insufficient. We suggest that the most suitable method for primary assessment of osteolytic involvement in monoclonal gammopathies should include either whole-body MRI together with CR of the skull or, with an equivalent sensitivity, whole body LD-CT.
\end{abstract}

Key words: multiple myeloma, monoclonal gammopathy of undetermined significance, imaging methods, conventional radiography, whole-body magnetic resonance, whole-body low-dose computed tomography

Received: May 29, 2015; Accepted: December 3, 2015; Available online: January 5, 2016 http://dx.doi.org/10.5507/bp.2015.064

${ }^{a}$ Department of Hemato-Oncology, University Hospital Olomouc and Faculty of Medicine and Dentistry, Palacky University Olomouc, Czech Republic

${ }^{b}$ Department of Radiology, University Hospital Olomouc and Faculty of Medicine and Dentistry, Palacky University Olomouc, Czech Republic Corresponding author: Jiri Minarik, email: abretina@email.cz

\section{INTRODUCTION}

Multiple myeloma (MM) is a hematological malignancy characterized by proliferation of neoplastically transformed plasma cells with production of monoclonal immunoglobulin (M-protein) detectable in serum and/ or urine of patients. Most patients with newly diagnosed MM present with various extent of bone involvement in the form of myeloma bone disease (MBD). MBD is present in $80-90 \%$ of MM patients, and it is a major cause of morbidity and mortality ${ }^{1}$. Assessment of MBD is crucial for the diagnosis of multiple myeloma requiring therapy, and for prognosis and risk assessment ${ }^{1-5}$.

Although there are many new imaging techniques such as computed tomography (CT), magnetic resonance imaging (MRI) or positron emission tomography (PET), conventional radiography $(\mathrm{CR})$ has been for long considered to be the "gold standard" in majority of individuals with newly diagnosed monoclonal gammopathies. Several papers reported on its limited sensitivity and the need for more sophisticated imaging ${ }^{6-10}$. Until 2014, the guidelines of the International Myeloma Working Group (IMWG) acknowledged the benefit of modern imaging, especially of MRI but recommended it only in specific situations such as in "MM patients with normal conventional radiography and in all patients with apparently solitary plasmocytoma of bone", and as an urgent procedure in the suspicion of cord compression ${ }^{1}$.

In 2014, new IMWG guidelines for the diagnosis and treatment of multiple myeloma were published, moving the diagnostics of active MM to earlier phase ${ }^{11}$. Three new myeloma related events (MRE) have been stated that indicate high risk of organ involvement and the need for immediate treatment even before the development of endorgan damage. These three MREs are the bone marrow clonal plasma cell infiltration of $\geq 60 \%$, ratio of involved/ uninvolved free light chains $\geq 100$, and presence of $>1$ osteolytic lesion (more than $5 \mathrm{~mm}$ ) using MRI (ref. ${ }^{11}$ ). The guidelines indirectly exclude $\mathrm{CR}$ as the gold standard in MM and emphasize the utility of MRI imaging.

Regardless of the new guideliness, we concurrently carried out a prospective study in patients with monoclonal gammopathies to confirm that relying on $\mathrm{CR}$ is not only insufficient but may even underestimate the presence of MBD leading to imprecise diagnosis and delay of proper treatment. We compared the outcomes of three imaging techniques - conventional radiography (CR), low-dose computed tomography (LD-CT) and whole-body 
magnetic resonance imaging (WB-MRI) in a prospectively designed study evaluating sensitivity, availability and cost effectiveness of each method, and to answer whether conventional radiography should still be a part of the assessment of MBD.

\section{PATIENTS AND METHODS}

We designed a prospective study aimed at imaging techniques in patients with monoclonal gammopathies. The study was approved by local as well as government authorities and by the local Ethics Committee. Patients with newly diagnosed monoclonal gammopathies or patients with relapse/progression of MM were included in the study following their written informed consent. The patients were examined using imaging techniques - conventional radiography, LD-CT and WB-MRI. All patients were also assessed by densitometry which will be discussed elsewhere. Imaging using PET/CT was not included in this study as it would not be ethical to expose patients to further imaging method with significant radiation exposure, and as it is usually not accessible for routine practice.

Between June 2013 and December 2014 we assessed a cohort of 112 individuals with monoclonal gammopathies - 83 patients with MM and 28 individuals with MGUS. The patients were diagnosed according to standard IMWG criteria and signed an informed consent with the participation in the study. All subjects were 18 years or older with measurable serum or urine levels of M-protein. The baseline characteristics of the group correspond to standard representation of monoclonal gammopathies in Caucasian populations. The $\mathrm{M} / \mathrm{F}$ ratio was $1.1: 1$ with a median age 69 years (36-88 years), with common representation of immunohistochemical types as well as kappa : lambda ratio and International Staging System (ISS) staging.

We assessed the presence of MBD using CR, LD-CT and WB-MRI. Conventional radiography included chest, skull, pelvis, humerus and femur projections, two projections of cervical, thoracic, lumbar and sacral spine. Whole-body LD-CT was performed using a standard CT machine with lower radiation dose achieved by a manual reduction of voltage ( $50 \mathrm{~mA}$ and $120 \mathrm{kV}$ ); the total dose delivered to each patient was $\sim 4 \mathrm{mSv}$. Whole-body MRI was performed using a 1.5-T system (Siemens Magnetom Avanto, Erlangen, Germany). No IV contrast material was administered. In order to maintain invariable conditions and to avoid description bias based on knowledge of the other whole-body technique, the LD-CT method was assessed in all patients by one designated radiologist, and MRI was assessed by a different radiologist. In the case of differing description, the imaging techniques were reassessed and directly compared by one radiologist.

In all CR, LD-CT and WB-MRI we evaluated the presence of none, one, two, three, or more than three osteolytic lesions, the presence of pathological fractures, and the presence of extramedullary masses. The regions for comparison were the skull, spine and chest, pelvis, and "long bones" (humerus and femur). Separately we compared the concordance of the presence of pathological fractures and extramedullary masses. We compared the difference in the number and extent of osteolytic involvement, and especially the finding in CR negative patients.

\section{RESULTS}

Direct comparison of all three methods (CR, LD-CT and WB-MRI) was possible in 43 patients. In the remaining 69 we performed CR with either WB-MRI or LD-CT. When assessing the skull the findings of CR and LD-CT were in concordance in 78\%. LD-CT was slightly more sensitive than CR (showing more osteolytic lesions), moreover, it showed even osteolytic involvement of the jaws which was not distinguishable on CR (Fig. 1). There were no patients with positive finding on $C R$ and negative LDCT, however, there were $16 \%$ of patients with presence of osteolytic lesions on LD-CT with negative CR findings. When comparing LD-CT and WB-MRI the findings were identical in $59 \%$ of the cases. LD-CT was more sensitive than WB-MRI - in 27\% of cases LD-CT displayed the presence of osteolytic lesions despite WB-MRI negativity. Surprisingly, CR showed more skull lesions than WB-MRI.

When assessing the spine, concordance of CR and LD-CT was seen in $27 \%$, all the corresponding cases being "negative". There were $64 \%$ patients with more lesions on LD-CT compared to CR and in $24 \%$ of the assessed patients there was osteolytic involvement on LD-CT which was not seen on CR. WB-MRI found osteolytic involvement despite CR negativity in $59 \%$ of patients. Comparing

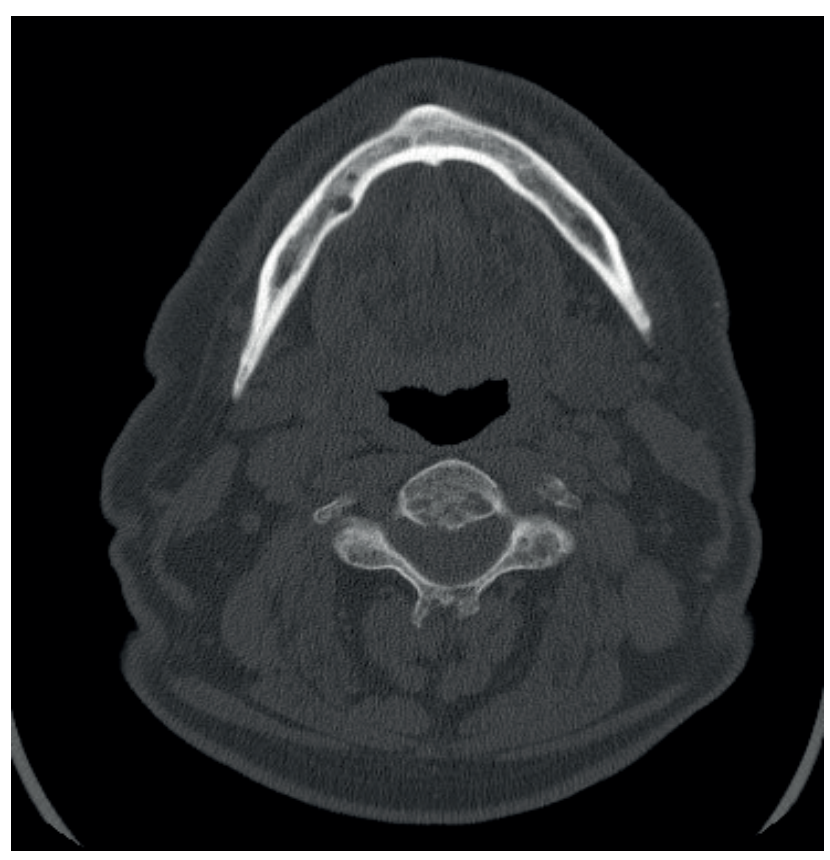

Fig. 1. The presence of osteolytic lesions in the jawbone in a patient with multiple myeloma using low-dose computed tomography. 
of LD-CT and WB-MRI in the spine showed higher sensitivity of WB-MRI. In 59\% the findings were identical, in $35 \%$ cases WB-MRI showed more osteolytic lesions, and in 6\% LD-CT showed more lesions. There were no patients with LD-CT positivity and WB-MRI negativity, however, 4 patients (23\%) with WB-MRI positivity had negative LD-CT scan. In one patient, the use of WB-MRI and LD-CT changed the diagnosis of monoclonal gammopathy of undetermined significance (MGUS) into active multiple myeloma with extramedullary involvement despite negative CR finding ${ }^{12}$.

In the chest, pelvis, humeri and femori, all three methods gave similar results with high concordance. In $15 \%$ of patients LD-CT and WB-MRI showed osteolytic involvement despite negative CR findings.

We found pathological fractures in $38 \%$ of the patients. The fractures were confirmed by all, conventional radiography, LD-CT and WB-MRI. Extramedullary masses were detected in $24 \%$ of patients. CR revealed one case only, which was also palpable on a thickened rib. LD-CT found all masses except one - where there was an extramedullary mass in the spinal canal which did not disrupt corticalis of the vertebral column, and it was therefore not visible using LD-CT (Fig. 2). WB-MRI also found all masses except for one which was out of the assessed field. In one case there was an extramedullary mass described on WB-MRI which turned out to be a false positive due to not very clear finding at the rim of the assessed section.

In patients with monoclonal gammopathy of undetermined significance $(\mathrm{N}=29)$ we expected no osteolytic involvement. However, in one patient (3\%) we found osteolytic lesions with extramedullary involvement changing the diagnosis into active MM (ref. ${ }^{12}$ ). In 3 patients (10\%) on CR and in 4 patients (14\%) on LD-CT we found osteolytic lesions despite these patients were diagnosed with MGUS. All the lesions were present either in the skull or pelvis. More than 1 lesion was present in 3 individuals (10\%). Within the 2-year follow-up, none of these individuals developed MM but all of them are being carefully observed.

\section{DISCUSSION}

Myeloma bone disease (MBD) is one of the most frequent manifestations of MM. Based on radiological studies, it is present in up to $80 \%$ of patients with newly diagnosed MM (ref. ${ }^{1,2,4,13-16}$ ). The other symptoms of MM are found less frequently - approximately $20 \%$ of patients develop hypercalcemia and $20-25 \%$ have renal impairment, anemia is present usually in $\sim 60 \%$ of patients and might be attributable to conditions other than myeloma. Therefore, MBD is the leading symptom of MM and should be carefully addressed with regard to the principal decision to treat or not to treat. Typical osteolytic lesions lead to pain, eventually to the involvement of vertebrae with pathological fractures and spinal cord compression. Clinical impact of MBD significantly influences both overall survival and the quality of life of patients with MM (ref. ${ }^{17}$ ). Patients with pathological fractures have more than $20 \%$ higher risk of death compared to patients without fractures ${ }^{18}$

Evaluation of the presence of osteolytic involvement is therefore one of the crucial steps in the assessment of monoclonal gammopathies, and it is the cornerstone of MM diagnostics and prognostication. Until now, there
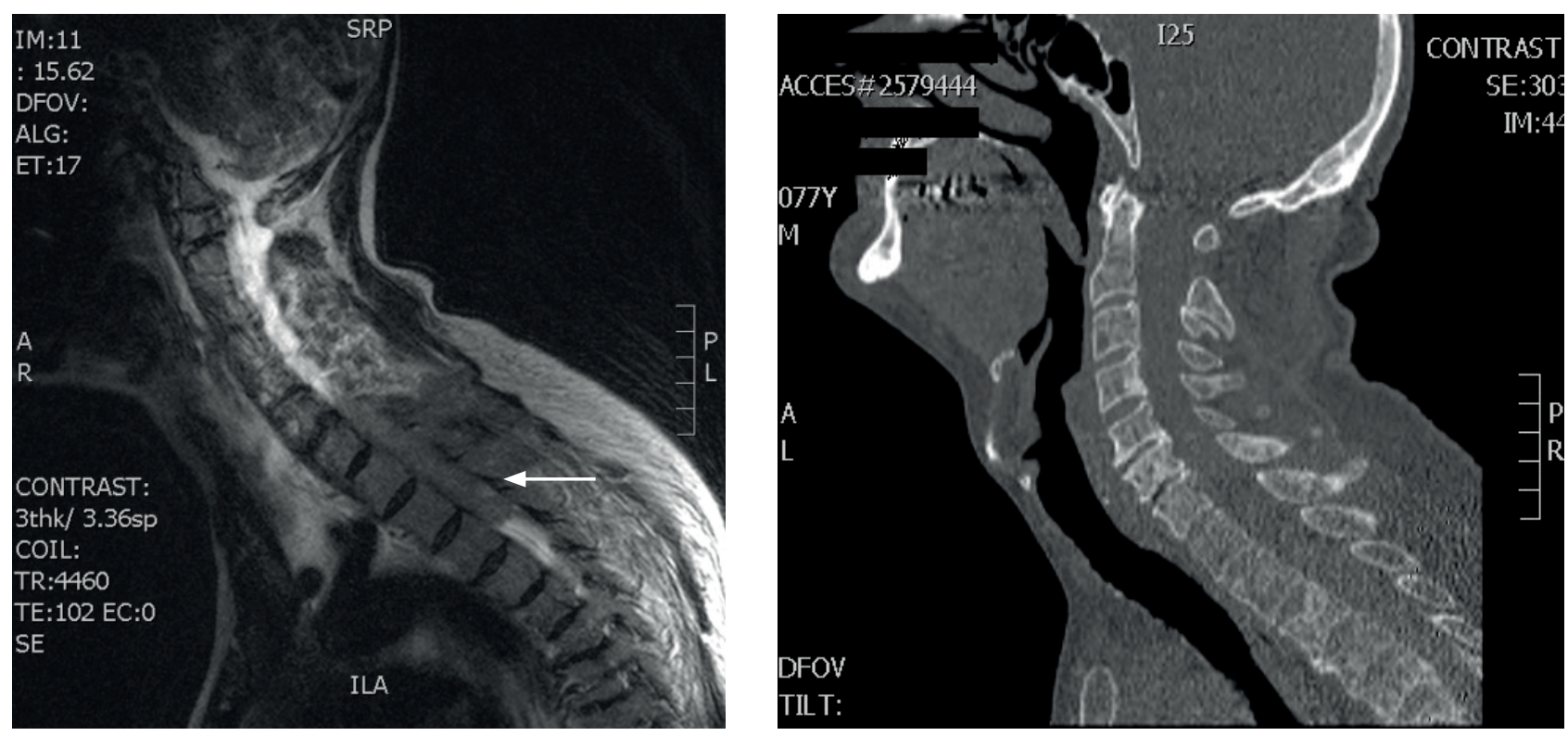

Fig. 2. Extramedullary lesion in the spinal column of a patient with multiple myeloma - comparison of low-dose computed tomography and magnetic resonance imaging.

Magnetic resonance imaging (left) showing in the STIR sequece a large extramedullary lesion (white arrow) in the spinal column of a patient with multiple myeloma.

Low-dose computed tomography (right) of the same region showed more osteolytic lesions of vertebrae, however, it did not show the extramedullary lesion as it did not disrupt the cortical bone. 
have been only a few papers prospectively comparing the role of imaging methods in MM (ref. ${ }^{8-10}$ ). Most of the data come from retrospective analyses or from studies aimed at one imaging method only. Modern imaging methods (such as MRI, CT, PET/CT) are significantly more sensitive and specific than CR. Still, there is no clear recommendation that would extricate the diagnostics of MM from the rather outdated CR. While the 2003 IMWG guidelines emphasized WB-MRI, still, they only recommended it for specific situations, such as solitary plasmocytoma or MM without the presence of osteolytic lesions on conventional radiography ${ }^{1}$. This approach is cost-saving, however, it may result in underestimation of the extent of MBD, or even missing the correct diagnosis altogether, as seen in our previous observation ${ }^{12}$. On the other hand, asymptomatic skeletal lesions detected only by MRI, CT or PET were not considered as routine indications for therapy in the recommendations for MM diagnosis by Kyle and Rajkumar in 2009 (ref. ${ }^{19}$ ).

Based on current knowledge, conventional radiography might have up to $30-70 \%$ false negative findings rate in the spine which is the most frequent localization of pain ${ }^{2}$. Our findings support this observation - in our study, CR did not show superiority in any of the assessed regions, ie. skull, pelvis, chest, humeri and femori; and in spine it failed, having $27 \%$ negative findings in comparison with LD-CT and up to $59 \%$ negative findings in comparison with WB-MRI. Similarly, CR failed to detect extramedullary lesions, which were found on both, LD-CT and WB-MRI. These results suggest that relying solely on $\mathrm{CR}$ would lead to misdiagnosis of active MM requiring treatment in substantial portion of patients, and about quarter of patients would have unrecognized extramedullary plasmocytoma.

Current IMWG criteria for the diagnosis of multiple myeloma from 2014 significantly shifted the diagnosis and treatment of MM to early phases of the disease ${ }^{11}$. Three new biomarkers of malignancy were added defining patients requiring therapy despite the lack of "traditional" end organ involvement criteria such as hypercalcemia, renal failure, anemia or bone lesions on CR. These three new parameters include $\geq 60 \%$ bone marrow clonal plasma cells, involved : uninvolved serum free light chain ratio $\geq 100$, and $>1$ focal lesion (over $5 \mathrm{~mm}$ ) on MRI studies ${ }^{11}$. These patients are at high risk of progression to active MM within 2 years and should be therefore indicated for immediate therapy. The presence of $>1$ focal lesion on MRI unambiguously documents the need for better imaging than $\mathrm{CR}$ in the diagnosis of MM.

The question is whether MRI should be obligatory in newly diagnosed monoclonal gammopathies or whether it might be substituted with other imaging techniques. Some studies comparing WB-MRI and PET/CT showed that whole body MRI has higher sensitivity and specificity over PET/CT, which is more sensitive in the detection of extramedullary plasmocytoma ${ }^{1,3,4,20,21}$. MRI is able to distinguish tumorous and benign structural changes, and even to differentiate incipient MBD in the form of "pepper and salt" involvement from osteoporosis. Its advantage is also in the precise detection of extramedullary process- es including potential spinal cord compression. Our study confirmed the superiority of WB-MRI in the spine, and it had similar findings as CR and LD-CT in the chest, pelvis, humeri and femori. In the skull, however, MRI did not show up to $27 \%$ of osteolytic lesions clearly detected by LD-CT. The reason is probably due to the skull structure which differs from other bones, and also due to limited sensitivity of MRI in osseous material.

CT-based imaging provides better detection of skeletal involvement than both conventional radiography and MRI. Like MRI, it is able to detect extramedullary processes, and when combined with positron emission tomography (as PET/CT) it can assess the activity of the process $^{2-4,20}$. Due to high radiation exposure associated with CT scans, effort has focused at reducing it using "lowdose" schedules ${ }^{7-9,22}$. The final image of "low-dose" CT is not as sensitive for soft tissue visualisation but it retains sensitivity for detection of bone involvement. Aiming for the most suitable, economical and easily available technique for routine practice, we chose low-dose computed tomography out of the CT-based imaging techniques for our study. LD-CT is an easily accessible method (using an ordinary CT machine) with similar costs and just slightly elevated radiation exposure in comparison with conventional radiography ${ }^{6,7,22}$. As shown by the present paper, LD-CT is more sensitive than WB-MRI on the skull, and it has similar sensitivity on pelvis and "long bones" including extramedullary involvement. It does not reach the sensitivity of WB-MRI in the assessment of the spine, however, there were only 4 patients (23\%) with at least one spinal lesions on WB-MRI who had negative LD-CT scan of this region. Furthemore, this discrepancy between LD-CT and WB-MRI would not have changed disease classification in any of these patients. There was one notable pitfall of LD-CT imaging - it failed to find one serious extramedullary lesion inside the spinal canal that did not disrupt the corticalis of the bone (Fig. 2). On the other hand, LD-CT was substantially cheaper than WB-MRI, faster and more comfortable for the patients with no need for prolonged and painful manipulation, with just slightly increased radiation exposure compared to CR, and it could be used also in patients with the presence of metallic implants in whom MRI is contraindicated or inaccessible.

Unlike the recent IMWG recommendations, we did not exclude lesions under $5 \mathrm{~mm}$ (ref. ${ }^{11}$ ). The sensitivity of each technique is quite different in the assessment of bone involvement, and our former criteria in the prospective assessment did not include this condition. Based on the IMWG recommendations, we plan, however, to expand and re-assess the cohort so that the findings correspond to current definition of MM in order to define the percentage of patients who would be underdiagnosed by each technique. Similarly, we plan to expand and follow the group of MGUS individuals with the presence of any osteolytic involvement. Following re-assessment, only one of the MGUS individuals in our cohort had lesions over 5 $\mathrm{mm}$ and no one fulfilled any other criteria of MM suggesting that the described lesions might not be attributable to MBD or that they might have some prognostic potential for future development of MBD. 
Our paper supports the idea that CR is not sensitive enough in the assessment of MBD, and that the assessment of the spine and extramedullary lesions using CR is rather poor in comparison with modern imaging methods $^{1-4,13-16}$. Still, CR can be used for the detection of osteolytic lesions of the skull, pelvis, ribs and long bones where it has acceptable sensitivity. Our recommendations for suitable imaging based on the present study are following: Apart from urgent procedures which should follow the current guidelines, the best choice for MBD assessment in newly diagnosed monoclonal gammopathies should be whole-body magnetic resonance with CR of the skull, or, with an equivalent sensitivity, whole-body LD-CT, which should, in the case of diagnostic uncertainty, be supported by MRI of the spine.

\section{CONCLUSION}

We conclude that conventional radiography should no longer be the cornerstone of MBD diagnostics. In accordance with the IMWG recommendations, whole-body MRI should be the preferred method, however, it can be substituted with LD-CT. The results of WB-MRI and LDCT are similar, and they both reveal the extent of MBD and extramedullary involvement.

\section{Acknowledgement: With support of the grant IGA MZ} CR NT14393.

Author contributions: JM: manuscript writing; JH, MH performed the imaging techniques and revisions, JM, PK, TP, JB, VS: diagnosed and treated the patients, JM, PK: data analysis; TP, JB, VS critical revision of the manuscript.

Conflict of interest statement: The authors declare there are no conflicts of interest regarding the publication of this article.

\section{REFERENCES}

1. Dimopoulos M, Terpos E, Comenzo RL, Tosi P, Beksac M, Sezer O, Siegel D, Lokhorst H, Kumar S, Rajkumar SV, Niesvizky R, Moulopoulos LA, Durie BG. International myeloma working group consensus statement and guidelines regarding the current role of imaging techniques in the diagnosis and monitoring of multiple myeloma. Leukemia 2009;23:1545-56.

2. Terpos E, Moulopoulos LA, Dimopoulos MA. Advances in Imaging and the Management of Myeloma Bone Disease. J Clin Oncol 2011;29:1907-15.

3. Hanrahan ChJ, Christensen CR, Crim JR. Current concepts in the evaluation of multiple myeloma with MRI imaging and FDG PET/ CT. RadioGraphics 2010;30:127-42.

4. Lutje S, de Rooy JWJ, Croockewit S, Koedam E, Oyen WJG, Reinier A. Role of radiography, MRI and FDG-PET/CT in diagnosis, staging and therapeutical evaluation of patients with multiple myeloma. Ann Hematol 2009;88:1161-8.

5. Myslivecek M, Nekula J, Bacovsky J, Scudla V, Koranda P, Kaminek M. Multiple myeloma: predictive value of Tc-99m MIBI scintigraphy and MRI in its diagnosis and therapy. Nucl Med Rev 2008;11:91-5.

6. Delorme S, Baur-Melnyk A. Imaging in multiple myeloma. Eur J Radiol, 2009;70:401-8.
7. Mahnken AH, Wildberger JE, Gehbauer G, Schmitz-Rode T, Blaum M, Fabry U, Gunther RW. Multidetector CT of the spine in multiple myeloma: comparison with MR imaging and radiography. Am J Roentgenol 2002;178:1429-36.

8. Baur-Melnyk A, Buhman S, Becker C, Schoenberg SO, Lang N, Bartl $\mathrm{R}$, Reiser MF. Whole body MRI versus whole body MDCT for staging of multiple myeloma. Am J Roentgenol 2008;190:1097-104.

9. Gleeson TG, Moriarty J, Shortt CP, Gleeson JP, Fitzpatrick P, Byrne B, McHugh J, O'Connell M, O'Gorman P, Eustace SJ. Accuracy of whole-body low-dose multidetector CT (WBLDCT) versus skeletal survey in the detection of myelomatous lesions and correlation of disease distribution with whole-body MRI (WBMRI). Skeletal Radiol 2009;38:225-36.

10. Zamagni E, Nanni C, Patriarca F, Englaro E, Castellucci $P$, Geatti $O$, Tosi P, Tacchetti P, Cangini D, Perrone G, Ceccolini M, Brioli A, Buttignol S, Fanin R, Salizzoni E, Baccarani M, Fanti S, Cavo M. A prospective comparison of $18 \mathrm{~F}$-fluordeoxyglucose positron emission tomography-computed tomography, magnetic resonance imaging and whole-body planar radiographs in the assessment of bone disease in newly diagnosed multiple myeloma. Haematologica 2007;92:50-5.

11. Rajkumar SV, Dimopoulos MA, Palumbo A, Blade J, Merlini G, Mateos $\mathrm{M}-\mathrm{V}$, Kumar S, Hillengass J, Kastritis E, Richardson $\mathrm{P}$, Landgren $\mathrm{O}$, Paiva B, Dispenzieri A, Weiss B, LeLeu X, Zweegman S, Lonial S, Rosinol L, Zamagni E, Jagannath S, Sezer O, Kristinsson SY, Caers J, Usmani SZ, Lahuerta JJ, Johnsen HE, Beksac M, Cavo M, Goldschmidt $H$, Terpos E, Kyle RA, Anderson KC, Durie BGM, San Miguel JF. International Myeloma Working Group updated criteria for the diagnosis of multiple myeloma. Lancet Oncol 2014;15:538-48.

12. Minarik J, Hrbek J, Pika T, Novak M, Bacovsky J, Herman M, Hrabalek L, Frysakova L, Pusciznova P, Scudla V. X-ray in Multiple Myeloma - Not a "Golden Standard" any More: Case Series. J Bone Mar Res 2014;2:1-4. doi:10.4172/2329-8820.1000149.

13. Healy CF, Murray JG, Eustace SJ, Madewell J, O'Gorman PJ, O'Sullivan P. Multiple Myeloma: A Review of Imaging Features and Radiological Techniques. Bone Mar Res 2011, Article ID 583439. doi:10.1155/2011/583439.

14. Zamagni $E$, Cavo M. The role of imaging techniques in the management of multiple myeloma. Brit J Haematol 2012;159:499-513.

15. Walker RC, Brown TL, Jones-Jackson LB, De Blanche L, Bartel T. Imaging of multiple myeloma and related plasma cell dyscrasias. J Nucl Med 2012;53:1091-101.

16. Regelink JC, Minnema MC, Terpos E, Kamphuis MH, Raijmakers PG, Pietes van den Bos IC, Heggelman BG, Nievelstein RJ, Otten RH, van Lammeren-Venema D, Zijlstra JM, Arens Al, de Rooy JW, Hoekstra OS, Raymakers R, Sonneveld P, Ostelo RW, Zweegman S. Comparison of modern and conventional imaging techniques in establishing multiple myeloma-related bone disease: a systemic review. Brit J Haematol 2013;162:50-61.

17. Cocks K, Cohen D, Wisløff F, Sezer O, Lee S, Hippe E, Gimsing P, Turesson I, Hajek R, Smith A, Graham L, Phillips A, Stead M, Velikova G, Brown J, EORTC Quality of Life Group. An international field study of the reliability and validity of a disease-specific questionnaire module (the QLQ-MY20) in assessing the quality of life of patients with multiple myeloma. Eur J Cancer 2007;43:1670-8.

18. Saad F, Lipton A, Cook R, Chen YM, Smith M, Coleman R. Pathologic fractures correlate with reduced survival in patients with malignant bone disease. Cancer 2007;110:1860-7.

19. Kyle RA, Rajkumar SV. Criteria for diagnosis, staging, risk stratification and response assessment of multiple myeloma. Leukemia 2009;23:39.

20. Shortt CP, Gleeson TG, Breen KA, McHugh J, O'Connell MJ, O'Gorman PJ, Eustace SJ. Whole-Body MRI versus PET in assessment of multiple myeloma disease activity. Am J Roentgenol 2009;192:980-6.

21. Hillengas J, Weber M-A, Kilk K, Listl K, Wagner-Gund B, Hillengass M, Hielscher T, Farid A, Neben K, Delorme S, Landgren O, Goldschmidt $\mathrm{H}$. Prognostic significance of whole-body MRI in patients with monoclonal gammopathy of undetermined significance. Leukemia 2014;28:174-8.

22. Ippolito D, Besostri V, Bonaffini PA, Rossini F, Di Lelio A, Sironi S. Diagnostic value of whole-body low-dose computed tomography (WBLDCT) in bone lesions detection in patients with multiple myeloma (MM). Eur J Radiol 2013,82:2322-7. 